\title{
COMPARATIVE STUDY BETWEEN THREE DIFFERENT PLATING TECHNIQUES IN MANAGEMENT OF MANDIBULAR ANGULAR FRACTURES. A RETROSPECTIVE STUDY
}

\author{
Nermine Ramadan Mahmoud * and Essam M. Ashour**
}

\begin{abstract}
Aim of Study: was to compare both clinically and radiographically between the threedimensional plates, 2.4 reconstruction plates and superior border Champy's single miniplate fixation in the treatment of mandibular angle fractures.

Materials and Methods: The study enrolled patients who suffered from mandibular angle fractures, presented to the outpatient clinic of the Oral and Maxillofacial Surgery department, Faculty of Dentistry, October 6 University as well as to the emergency unit of October 6 University Hospital. Patients were divided into 3 equal groups; Group I: comprised of 10 patients where ORIF through intraoral approach using three Dimensional miniplates assisted by trans-buccal trocar was done. Group II: comprised of 10 patients where ORIF through extraoral approach using 2.4 Reconstruction plate was done. Group III: comprised of 10 patients where ORIF through intraoral approach using single superior border miniplates (Champy's technique) was done.

Follow up were scheduled 1 week then 1,3 and 6 months post-operatively. Intra-operative outcomes included: ease of accessibility, ease of adaptation, need for post-operative IMF and approach related complications. Post-operative clinical findings were obtained along the follow up period. All patients were evaluated for maximum interincisal opening. Maximum intercuspal position to assure the midline centralization and the proper occlusal relationship including molar relation. Any occlusion disturbance, premature contact or open bite was considered as malocclusion. Sensory and motor nerve function was assessed along the follow up period as well as mandibular segment stability. Panoramic x-ray was done during the first week postoperatively to evaluate reduction \& fixation of the fractured segment, then periodically in each follow-up visit.
\end{abstract}

Results: Statistical analysis of this study concluded that (Group I) presented statistically significant lower infection rate and lower possibility of sensory and motor nerve affection (Group I) and (Group II) presented proper occlusion, more segment stability and fracture rigidity in the treatment of mandibular angular fractures in comparison to (Group III).

\footnotetext{
* Lecturer at Oral and Maxillofacial Surgery Department, Faculty of Dentistry, October 6 University, Egypt

** Associated Professor at Oral and Maxillofacial Surgery Department, Faculty of Dentistry, October 6 University, Egypt
} 
Conclusion: The 3D plates demonstrated a superior advantage over the 2.4 Reconstruction plates of ease of insertion transbuccally avoiding any extraoral cutaneous scars, lower infection rates and negligible possibility of facial nerve affection. Moreover, it obviates the need for immobilization of the mandible as required in Champy's technique.

KEYWORDS: mandibular angle fractures; Transbuccal trocar; 3D plates; 2.4 reconstruction plate; champy's technique.

\section{INTRODUCTION}

Road traffic accidents considered the most common etiology among the causes of maxillofacial fractures.(Ahmed,2019) Management of mandibular fractures has been promoted from intermaxillary fixation (IMF) to a combination of IMF and wire osteosynthesis, lag screw, and plate fixation. Despite the development in techniques, open reduction and internal fixation (ORIF) were considered the commonest treatment modality regarding each case condition and severity and has proven to be completely freed from major complications. As well as, ORIF offered many benefits outcomes over the closed reduction (Ahmed, 2019; Ravikumar et al., 2019)

Currently, many types of plating system are used such as the standard miniplates, reconstruction plates and the 3-dimensional miniplates. The purpose of mandibular fracture treatment must aim to the perfection of proper anatomic reduction, fixation and stability. The use of 3-dimensional (3D) strut plates. The 3D plates with its box stable configuration is considered as a two-plate system that gives more support. (Kubila et al., 2017)

The aim of the current study was to compare both clinically and radiographically between the three-dimensional plates, the 2.4 reconstruction plates and the superior border Champy's single miniplate fixation in the treatment of mandibular angle fractures.

\section{MATERIALS AND METHODS}

This study was conducted on 30 patients who were selected in the Oral and Maxillofacial surgery department, Faculty of Dentistry, October 6 University, as well as to the emergency unit of October 6 University Hospital, Egypt. Patients suffering from mandibular angle fractures were included in this study, divided randomly into 3 equal groups. Group I: comprised of 10 patients where ORIF through intraoral approach using three Dimensional miniplates assisted by transbuccal trocar was done. Group II: comprised of 10 patients where ORIF through extraoral approach using 2.4 Reconstruction plate was done. Group III: comprised of 10 patients where ORIF through intraoral approach using single superior border miniplates (Champy's technique) was done.

Inclusion criteria: Patient's suffering from mandibular angle fracture, age ranges from 20-50 years, of both genders with displaced unilateral mandibular angle fractures.

Exclusion criteria: patients contraindicated to general anesthesia, patients with bone diseases affecting bone healing, patients presenting with infection at the fracture site and those not willing to return for follow up.

Before definitive treatment the following steps were performed: a standard panoramic view was taken for each patient at time of presentation (figures 1,2,3), other necessary views were requested according to each case as C.T scan, 3D CT. (figures 4,5)

Informed consent was obtained before surgical intervention. Care of any lacerated soft tissues and wound debridement for all patients presented with compound fractures. Intravenous antibiotics were administered preoperatively at the time of admission and were continued until discharge. 
Teeth in fracture line was managed according to preoperative assessment either by extraction or preservation. The patient was placed into maxillomandibular fixation with the aid of arch bars to achieved proper occlusion.

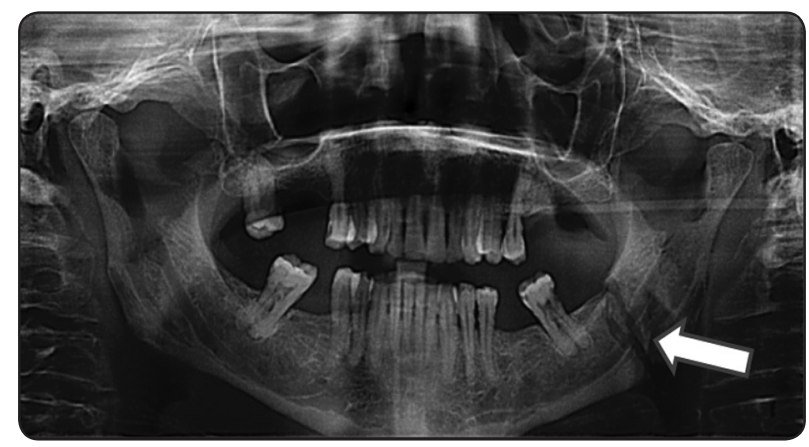

Fig. (1): Pre-operative panoramic radiograph showing left mandibular angle fracture in patient No 1 group I .

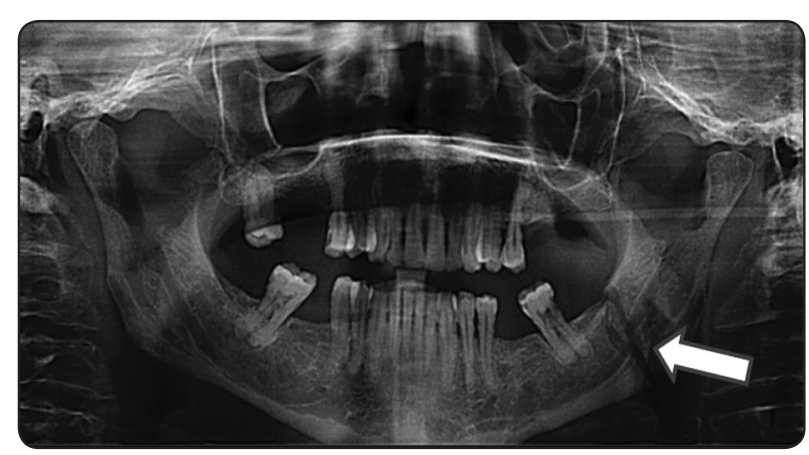

Fig. (2): Pre-operative panoramic radiograph showing left mandibular angle fracture in patient No 1 group III .

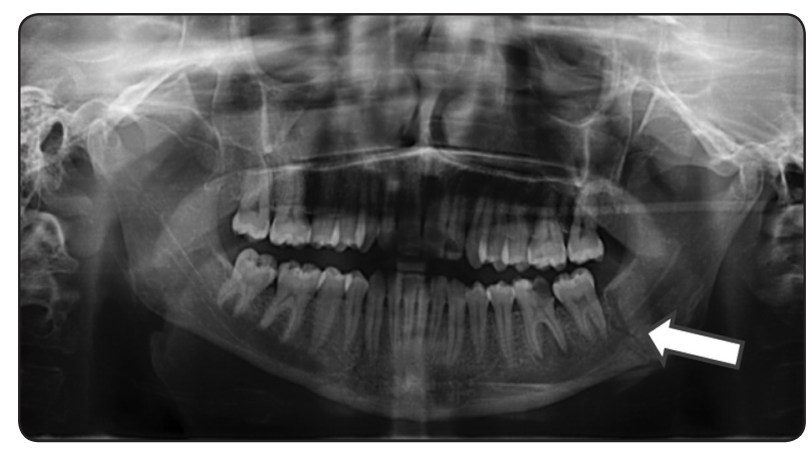

Fig. (3): Pre-operative panoramic radiograph showing left mandibular angle fracture in patient No 3 group III .

\section{Operative phase: Incisions/ Reduction of fracture}

The fracture site was exposed by a standard intraoral vestibular approach. A soft-tissue incision was then made over external oblique ridge, beginning midway up the anterior border of ascending ramus, and was carried into the vestibule just lateral to mandibular first molar. Subperiosteal dissection was then performed, exposing the lateral surface of the mandibular angle and ramus region extending to the posterior and inferior border. It is important to have adequate release of the tissue to allow visualization of the fracture.

J-stripper was used for releasing the PterygoMasseteric sling and anterior border stripper used for releasing of the temporalis tendon at the anterior border of the ramus with insertion of two channels under the inferior border of the proximal and distal segments for retraction and allowing maximum interdigitation and proper reduction of the fractured segments.

One small stab incision was made through the cutaneous surface, $1 \mathrm{~cm}$ below the inferior border. The stab incision was assisted by placing the index finger into the surgical site and pushing laterally over the desired site tenting the soft tissue. Then the trocar was inserted through the stab incision.

In group I, curved rectangular Titanium 3D miniplates* $(6 \times 2$ holes interconnected by vertical struts) of $2.0 \mathrm{~mm}$ thickness secured with titanium monocortical screws of $2.0 \mathrm{~mm}$ head diameter. The curved 3D miniplates were placed transorally at the middle of the buccal surface of the mandible across the reduced fractured segments and fixated with 2.0 $\mathrm{mm}$ monocortical screws by the aid of the percutaneously applied trans-buccal trocar. (figures 6,7)

Trans-buccal mono-cortical drilling was performed with irrigation followed by fixation with mono-cortical 2.0-mm screws measuring $5,7 \&$

\footnotetext{
* Jeil Medical Corporation, Guro-gu, Seoul, Korea
} 
$9 \mathrm{~mm}$ in length was done just through the lateral cortex, nearly the midway between the superior and the inferior borders of the mandible on the proximal or distal segment.

In group II, the angle was approached through a Risdon incision, 1/200,000 diluted adrenaline solution was injected subcutaneously for haemostasis. A curvilinear incision was carried out 2 fingers breadth below the inferior border of mandible in order to avoid injury to the facial nerve (the marginal mandibular branch). (figure 8)

Elevation of the subplatysmal flap was done. The facial artery and vein were identified and ligated. Blunt layered dissection was performed to approach

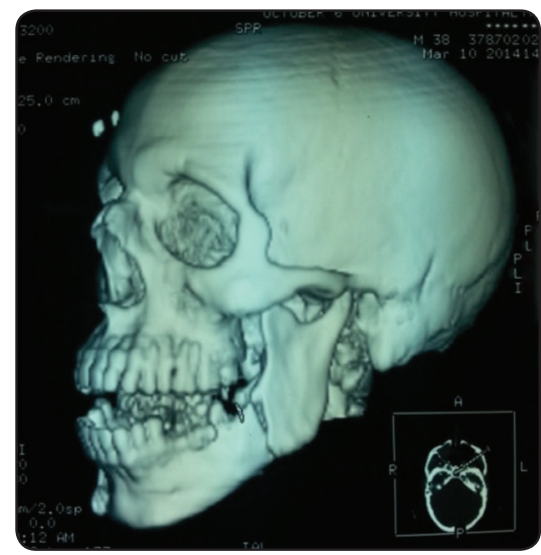

Fig. (4): Pre-operative 3D-CT radiograph showing left mandibular angle fracture in patient No 4 group II .

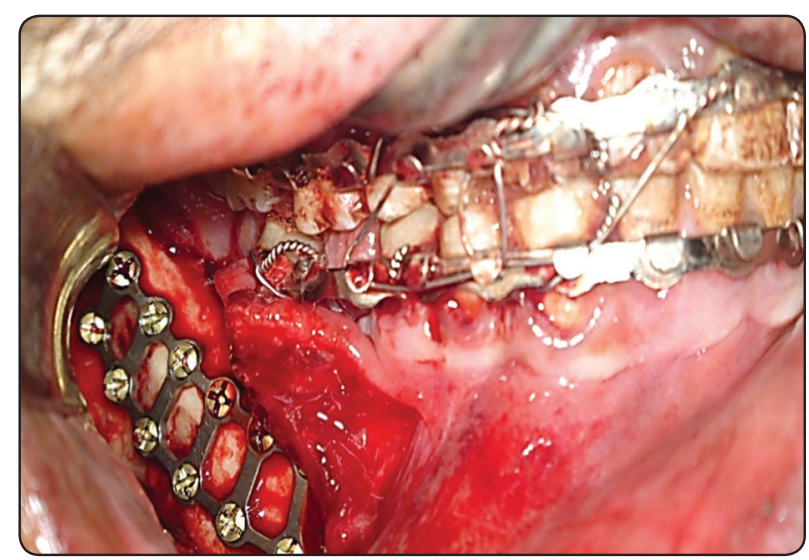

Fig. (6): 3D miniplate was fixated in right mandibular angular fracture in patient No 2 Group I. the mandibular angle after which the periosteum was incised at the inferior border of the mandible. Dissection of the pterygomassetric sling was done to expose the fractured bony segments. Bone clamps were used to approximate the fractured segments. (figure 9)

Then an angled 2.4 reconstruction plate was adapted and applied to bridge the fracture line and fix the reduced bony segments. (figure 10) Three screws on each side of the fracture line was used to fix the plate. The wound was closed in layers using $3 / 0$ vicryl sutures, the skin layer was closed with subcuticular 4/0 proline sutures. Surgical compression dressing was placed to prevent hematoma formation for approximately 24 hours.

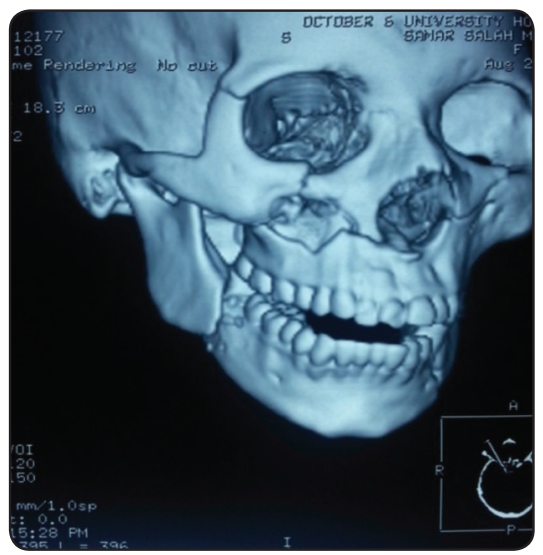

Fig. (5): Pre-operative 3D-CT radiograph showing right mandibular angle fracture in patient No 5 group I .

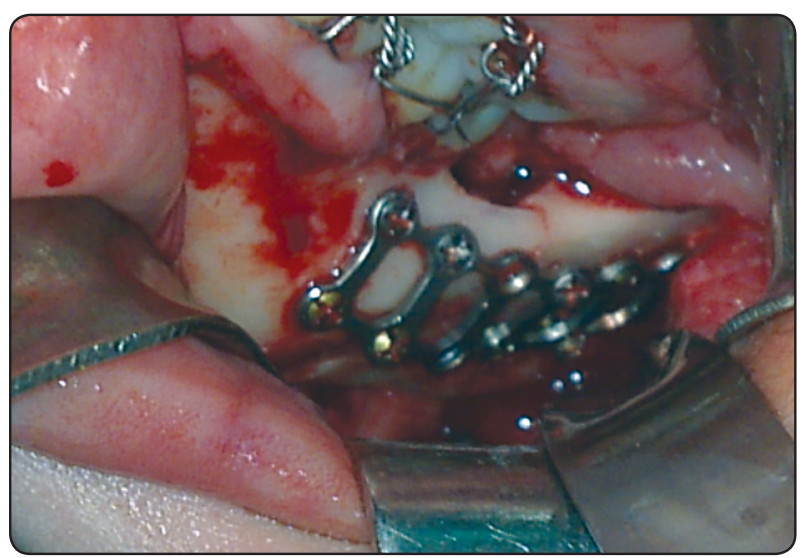

Fig. (7): 3D miniplate was fixated in left mandibular angular fracture in patient No 3 Group I. 
In group III (champy's technique), a six / eight holes monocortical miniplate was carefully contoured to superior buccal cortical area of mandible across the fracture line through an intraoral approach.

Reduction of fracture line was done by verifying alignment of buccal cortex and inferior border. Fixation was done by applying 6 or 8-hole conventional $2.0 \mathrm{~mm}$ titanium miniplate secured by titanium monocortical screws of $2.0 \mathrm{~mm}$ head diameter. 3 screws were inserted on either side to fix the plate. The miniplate must contact the bone surface throughout its length. (Figure 11)

Watertight wound closure was done. Upper third molar were evaluated to ensure it wouldn't traumatize the mucosal mandibular flap, if such trauma likely to occur, removal of upper wisdom tooth to prevent wound breakdown.

MMF was released and repeated occlusion was checked then the oral pack was removed. The skin layers were closed with sub-cuticular $4 / 0$ proline sutures. Surgical compression dressing was placed to prevent hematoma formation for approximately 24 hours. A soft diet was maintained for 4 weeks. Arch bars have been removed at 2 to 4 weeks.

Clinical and radiographic follow up were scheduled 1 week then 1,3 and 6 months postoperatively. Any complications were then recorded and treated. Intra-operative outcomes included: ease of accessibility, ease of adaptation, need for post-operative IMF and approach related complications. Post-operative clinical findings were obtained along the follow up period. All patients were evaluated for maximum interincisal opening. Maximum intercuspal position to assure the midline centralization and the proper occlusal relationship including molar relation. Any occlusion disturbance, premature contact or open bite was considered as malocclusion. Sensory and motor nerve function was assessed along the follow up period as well as mandibular segment stability. Panoramic x-ray was done during the first week postoperatively to evaluate reduction \& fixation of the fractured segment, then periodically in each follow-up visit.

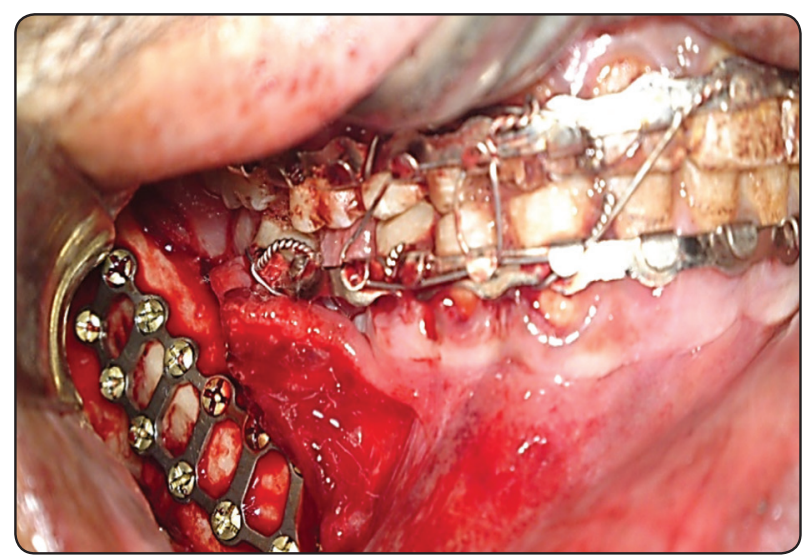

Fig. (8): Clinical photograph showing extraoral Risdon incision that was done in layers, 2 fingers breadth to inferior border of mandible to expose the right mandibular angle fracture in patient No 1 group II

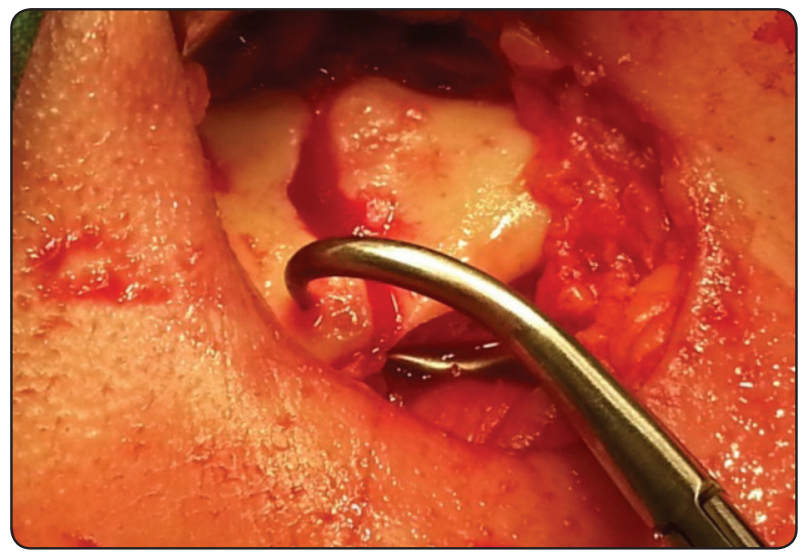

Fig. (9): Clinical photograph showing bone clamp used to approximate the fractured segments in patient No 1 group II .

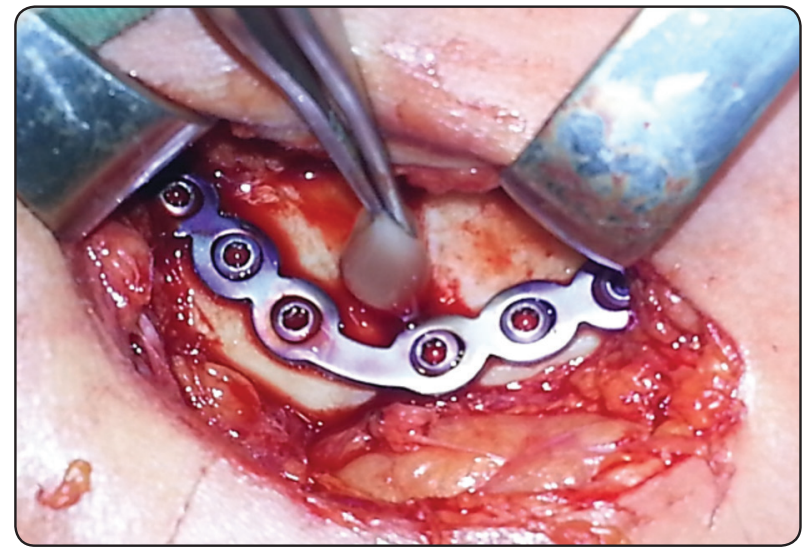

Fig. (10): Clinical photograph showing 2.4 reconstruction plate was applied to rigidly fix a right mandibular angular fracture in patient No 1 group II . 

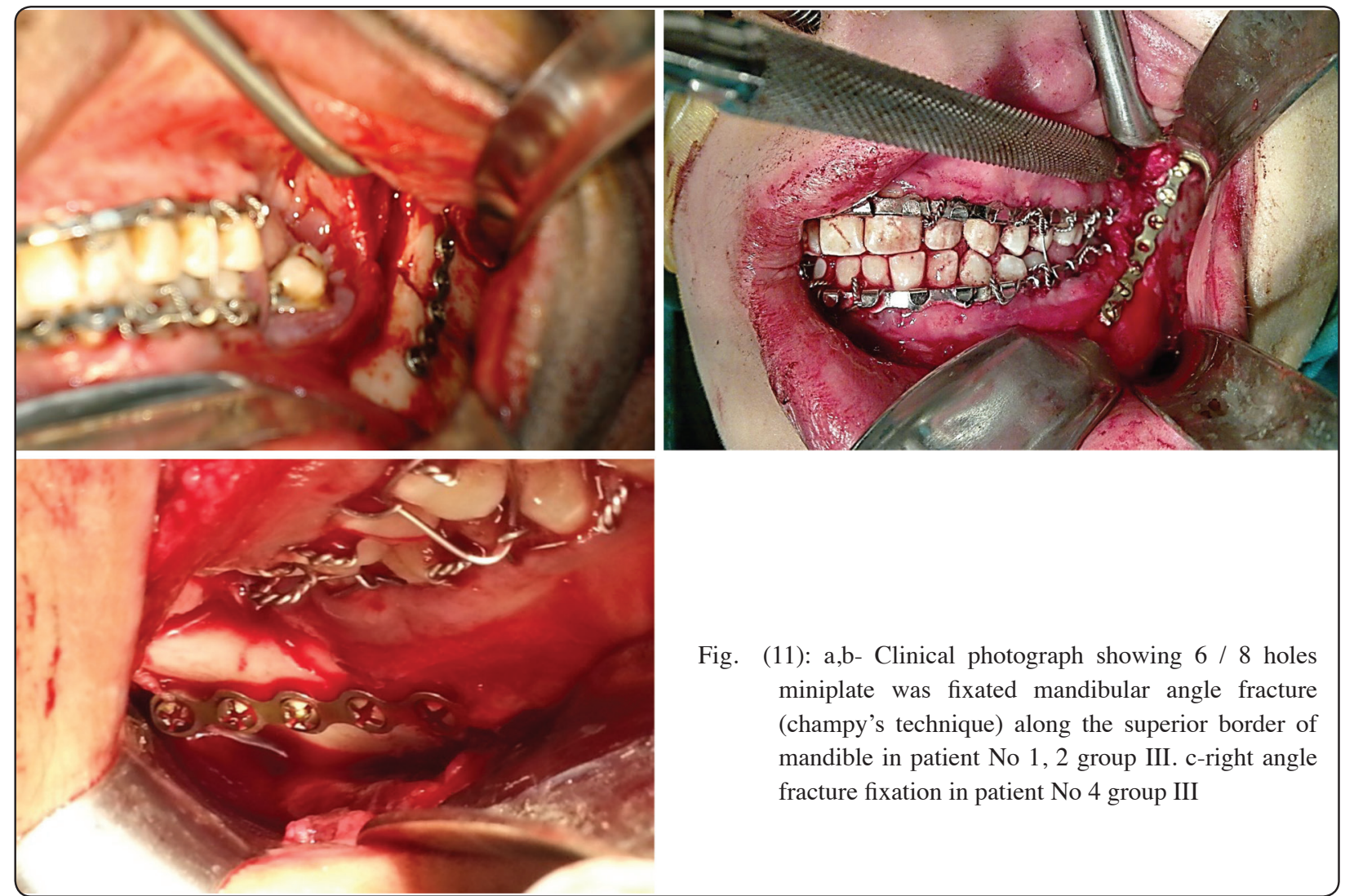

Fig. (11): a,b- Clinical photograph showing $6 / 8$ holes miniplate was fixated mandibular angle fracture (champy's technique) along the superior border of mandible in patient No 1, 2 group III. c-right angle fracture fixation in patient No 4 group III

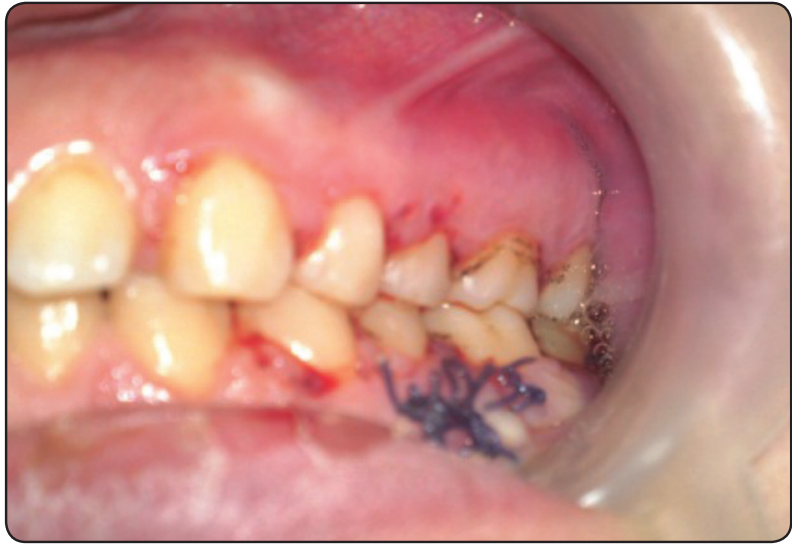

Fig. (12) : Clinical photograph showing post-operative healing

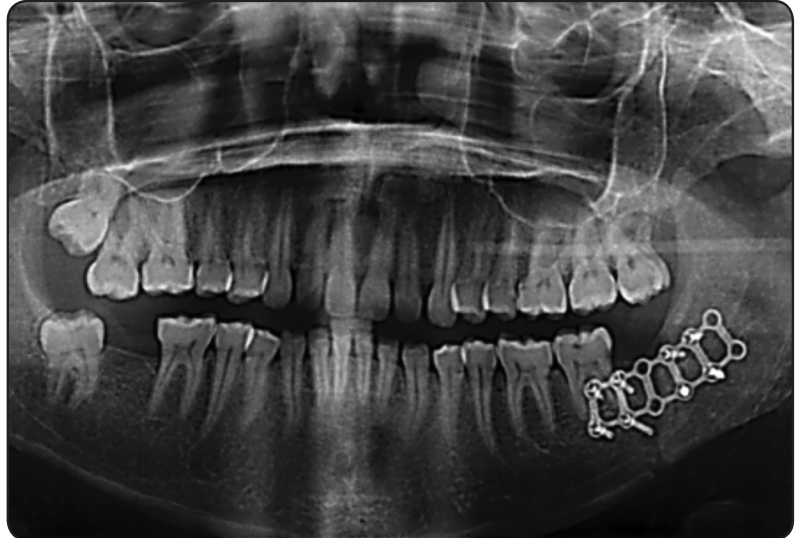

Fig. (13): Post-operative panoramic radiograph showing fixated left mandibular angle fracture with $3 \mathrm{~d}$ miniplate in patient No 1 group I . 


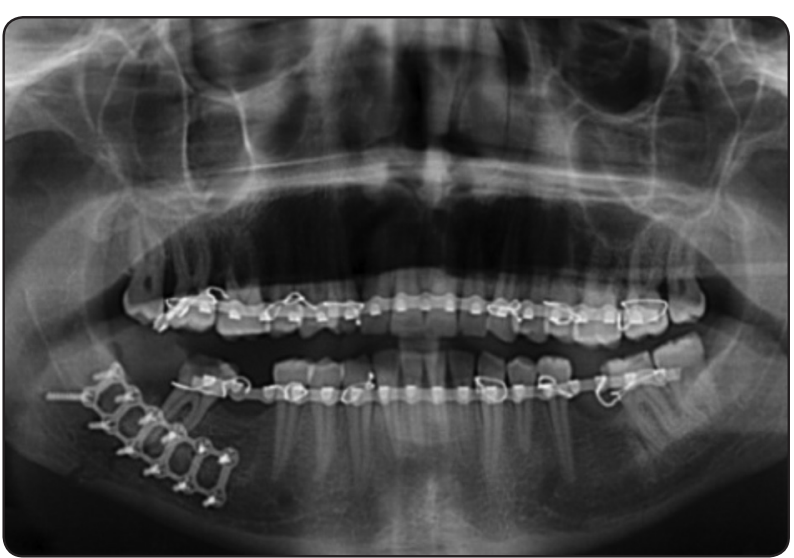

Fig. (14): Post-operative panoramic radiograph showing fixated right mandibular angle fracture with $3 \mathrm{~d}$ miniplate in patient No 2 group I .

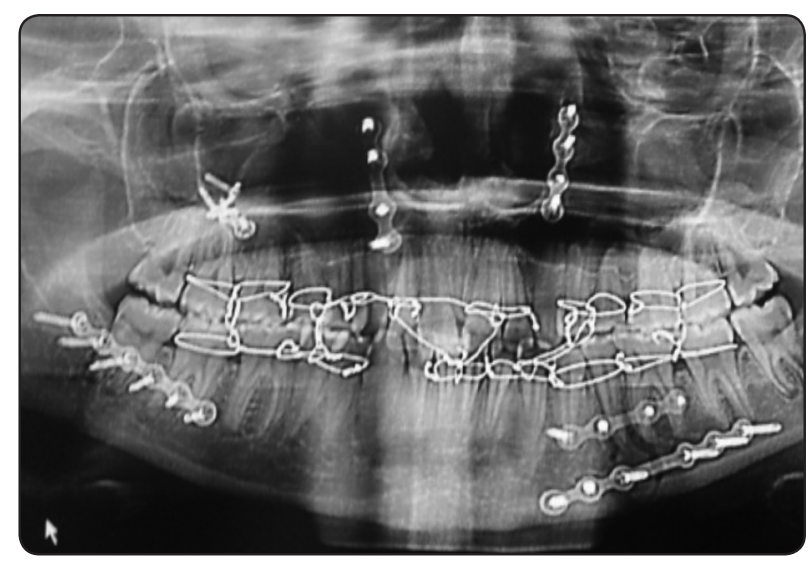

Fig. (15): Post-operative panoramic radiograph showing right mandibular angle fracture in patient No 6 group I .

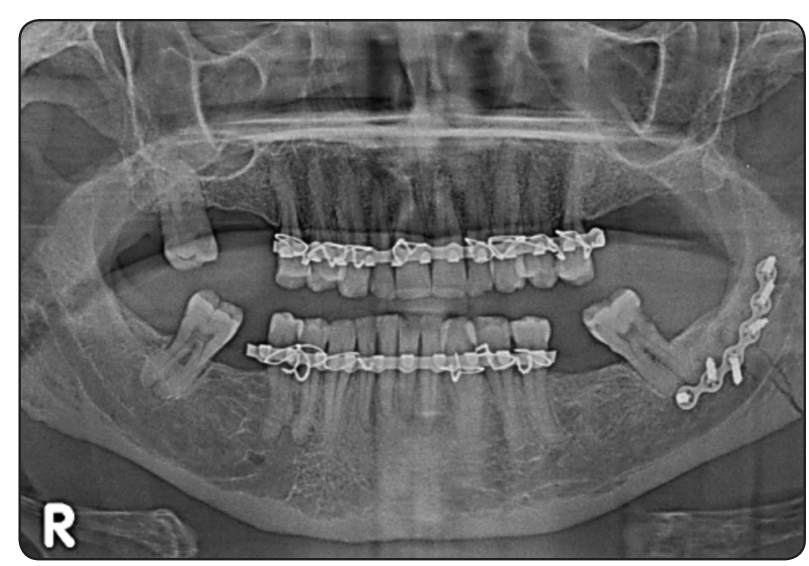

Fig. (16): Post-operative panoramic radiograph showing fixated left mandibular angle fracture in patient No 1 group III .

\section{RESULTS}

This study was designed to compare between three-dimensional plate, 2.4 reconstruction plate and single superior border miniplate fixation both clinically and radiographically, in treatment of mandibular angular fractures. All 30 patients enrolled completed the study. They ranged in age from 20 to 50 years with a mean age 31.3 years.

\section{Intra-operative Outcomes:}

Ease of accessibility: Intra-oral approach was considered as the easiest way to access the fracture line with the help of trans-buccal trocar. (Table 1) Accordingly, the Intra-oral approach was considered the easiest way to access the fracture line with the help of the trans-buccal trocar.

TABLE (1) : Showing the ease of accessibility intraoperatively

\begin{tabular}{|l|l|}
\hline $\mathbf{0}$ & No accessibility \\
\hline $\mathbf{1}$ & Good \\
\hline $\mathbf{2}$ & Fair \\
\hline $\mathbf{3}$ & Poor \\
\hline
\end{tabular}

Ease of adaptation of plate: The threedimensional plate was considered the easiest in point of view of its adaptation which return to its box configuration and no need for too much bending such that of 2.4 reconstruction plate which need more bending and adaptation on fractured bony segments.

Need for IMF post-operatively: This was considered as a case dependent according to segments displacement severity and time of patients presented to seek for treatments. That its delay lead to eburnation of the fractured bony edges with resultant fibrosis that in consequence lead to more difficult reduction and turning the fractured segments to its normal position 
Surgical time: It was measured from the start of the incision either intra or extra-oral to the end of tying the last suture with the same digital clock for all patients. The operative time ranged from $65-$ 110 min for group I (mean 83min), 105-145 min for group II (mean 125min), and 60-90 min for group III (mean 78min). Our study showed that surgical time needed in both group I and III using intra-oral sagittal split incisions is less than that of group II that needed extra-oral incision which required more surgical time and a higher operator expertise.

\section{Post-Operative Outcomes/ Clinical follow-up:}

Clinical evaluation was performed at 1 week and at 1,3 and 6 months postoperatively, and any complications were then recorded and treated. Occlusion was checked along the follow-up period. Maximum intercuspal position to assure the midline centralization and the proper occlusal relationship including molar relation. Any occlusion disturbance, premature contact or open bite was considered as malocclusion. Improper occlusion was found immediate post-operative in the $3 \mathrm{rd}$ and 5 th cases Group III. Heavy elastics and IMF were done for 14 days.

Sensory and motor nerve function: Sensory function was assessed along the follow up period; subjectively through asking the patient about any sensations' alteration in as well as objective examination by using a dental probe to assess the sensory changes along the distribution of the mental nerve (lower lip and chin) through examining lip sensation in comparison to the contralateral side. Motor nerve function was assessed by asking the patient to blow the cheek and to approximate both upper and lower lips and to smile widely while observing abnormal deviational movements of the lower lip at the ipsilateral side of suspected nerve injury.

One patient in Group I showed signs of paresthesia after the surgery, however, pre-operative numbness was detected clinically during preoperative examination. Paresthesia was still evident at the 3rd month interval and completely resolved at the 6th month follow-up period.

Three patients in Group II showed temporary weakness of the marginal mandibular branch of the facial nerve which gradually resolved throughout time and resolved completely at the $6^{\text {th }}$ month follow up period.

Maximum mouth opening: in mm using a polygauge on the second day, after the first week and after the first, third and six months postoperatively. The measurements were compared to that before the surgery.

Segment stability: All patients were checked along the follow-up period through bimanual palpation of mandibular segment to check stability. Assessment was done by applying bending and torsional forces across the fractured segments while detecting movements at the superior and inferior border as well as bucco-lingual splaying.

\section{Group I :}

At the first postoperative week, all patients were free from pain, edema, any sign of infection, wound dehiscence, with no segment mobility \& proper occlusion. (figure 12) The wound healing was uneventful for all patients with increasing interincisal opening.

After 2 years follow-up visit: the $4^{\text {th }}$ case in group I complained of pain \& screws sensation beneath the skin, on panoramic radiograph examination, it revealed loosening of screws and radiolucency's surrounded it. Patient underwent another surgical operation for plate and screws removal.

\section{Group II :}

All patients of this group exhibited perfect occlusion with no segment mobility throughout all the follow-up intervals. 
Occlusion and segment stability showed statistically significant higher values compared to group III while statistically insignificant higher values compared to group I.

Patients needed more restricted follow up for extra-oral wound care. The wound healing was uneventful for all patients with increasing in the interincisal opening. Extra-oral wound care was operator dependent during follow-up visits in this group.

\section{Group III :}

After 6 months, two patients presented with symptoms of pain and screw sensation which was confirmed radiographically by radiolucency around loose screws and clinically an intra-oral fistula was found at the left molar region which was managed by antibiotic course until the infection subsides and then screws and plate removal was done.

\section{Radiographic findings}

Panoramic x-ray was done during the first week postoperatively to evaluate reduction \& fixation of the fractured segment, then periodically in each follow-up visit. (figures 13-16) Data of both groups were tabulated and statistically analyzed.

Data of both groups were tabulated and statistically analyzed.

Comparison of wound dehiscence in 3D plates, 2.4 reconstruction plates and Champy's technique groups: Wound dehiscence was only observed in $20 \%$ of patients of Group III at the $1^{\text {st }}$ week immediately post-operative. No wound dehiscence could be detected in both groups in all other intervals. Using Fisher' exact test, the difference between both groups was found to be highly statistically significant at the immediate post-operative period. (Table 2)

Comparison of infection in 3D plates, 2.4 reconstruction plates and Champy's technique groups: Infection was only observed in $20 \%$ of patients of group III, 6 months post-operatively and in group I in the 2 years follow up. No infection could be detected in both groups in all other intervals. Using Fisher exact test, the difference between both groups was found to be highly statistically significant at the 6 months post-operative period. No statistically significant difference between all groups at all other follow-up intervals.

Comparison of segment stability in 3D plates, 2.4 reconstruction plates and Champy's technique groups: Preoperatively Fracture segments was mobile in all patients in all groups, while postoperatively it was evident in 3 patients of group III, however segment stability in all patients of groups I and II. At the first month of the follow up period and by using Fisher exact test, Group II showed statistically significant higher values of segment stability compared to group III while statistically insignificant higher values compared to group I. No segment mobility could be detected in both groups at all other follow-up intervals, with no significant difference.

TABLE (2) Percentage of wound dehiscence in both groups in different intervals

\begin{tabular}{|c|c|c|c|c|}
\hline & 3D plates & $\begin{array}{c}\mathbf{2 . 4} \text { reconstruction } \\
\text { plate }\end{array}$ & $\begin{array}{c}\text { Champy's } \\
\text { technique }\end{array}$ & $\begin{array}{c}\text { Statistical Significance } \\
\text { (p value) }\end{array}$ \\
\hline Immediate post operative & $0 \%$ & $0 \%$ & $20 \%$ & $<0.0001 * *$ \\
\hline $\mathbf{1}$ month post operative & $0 \%$ & $0 \%$ & $0 \%$ & Not significant \\
\hline $\mathbf{3}$ months post operative & $0 \%$ & $0 \%$ & $0 \%$ & Not significant \\
\hline $\mathbf{6}$ months post operative & $0 \%$ & $0 \%$ & $0 \%$ & Not significant \\
\hline
\end{tabular}

** highly statistically significant 
TABLE (3) Percentage of degree of displacement in the pre-operative observation period in 3D plates and Champy's technique groups

\begin{tabular}{|c|c|c|c|c|c|}
\hline & No displ & Satisfactory & Minimum displ & Moderate displ & severe displ \\
\hline 3 D plates & $0 \%$ & $0 \%$ & $40 \%$ & $0 \%$ & $60 \%$ \\
\hline Champy's & $20 \%$ & $0 \%$ & $20 \%$ & $40 \%$ & $20 \%$ \\
\hline
\end{tabular}

Statistical

Significance (p-value)

** highly statistically significant $<0.0001$

Comparison of proper occlusion in 3D plates, 2.4 reconstruction plates and Champy's technique groups: Excluding edentulous patients, proper occlusion could be detected in both groups in all intervals, except for one month interval of group III where four patients (40\%) presented with slight derangement of occlusion in the form of mild occlusal open bite, which necessitated the application of heavy elastics for 1 month to achieve an accepted repeatable occlusion. Using Fisher exact test, Groups I and II showed statistically significant higher values of proper occlusion compared to group III at one-month interval while also showed higher values of proper occlusion at other follow-up intervals although it was not statistically significant.

Comparison in incidence of paresthesia in 3D plates, 2.4 reconstruction plates and Champy's technique groups: One patient in group I showed signs of parasthesia after the surgery, however, preoperative numbness was detected clinically during pre-operative examination. Parasthesia was still evident at the $3^{\text {rd }}$ month interval and completely resolved at the $6^{\text {th }}$ month follow-up period. In group II, three patients showed temporary weakness of the marginal mandibular branch of the facial nerve which gradually resolved throughout time and cured completely at the $6^{\text {th }}$ month follow-up period.

Comparison of increase in inter-incisal
$<0.0001^{* *}$

opening in 3D plates, 2.4 reconstruction plates and Champy's technique groups: A progressive increase in the inter-incisal opening was observed in both groups at all follow-up intervals. Using Student's test, no significant difference could be detected between both groups regarding this increase.

Comparison of displacement in 3D plates, 2.4 reconstruction plates and Champy's technique groups: In the pre-operative observation period, severe displacement was observed in $60 \%$ of patients of the champy's technique, compared to $20 \%$ in patients of the 3D plates. Using chi-square test, the difference in displacement was highly statistically significant between both groups only in the pre-operative. In the subsequent intervals, the displacement was satisfactory in both groups, with no significant difference. (table 3 )

\section{DISCUSSION}

The aim of our study was to compare both clinically and radiographically between the threedimensional plates, the 2.4 reconstruction plates and the single superior border Champy's miniplate fixation in treatment of mandibular angle fractures.

The transbuccal trocar was considered a useful adjunctive instrument for accessing the fracture in the mandibular angle region. A transoral approach 
alone does not always provide adequate access to posterior fracture sites. This was particularly true if the surgeon wishes to fixate a posterior body or ramus fracture and seeking for avoiding a facial incision. (Thomas et al., 2009)

(Omezli et al., 2017) in their study on the biomechanical behaviors of two different types of osteosynthesis transbuccal and transoral that were used in the treatment of mandibular angle fractures, it was considered the peak loads and displacement values, they found no statistically significant differences between both groups.

In our study, we used transbuccal trocar in group III, in order to provide better accessibility to the most distal screws in the plates during fixation the reduced fractured segments.

Decision regarding the approach and their benefits outcomes either extra-oral or trans-oral approaches most often depended upon the anatomical location of the fracture line, type of fracture, amount of displacement of fractured segments, dentition of the patient, associated maxillofacial fractures and general condition of the patient. (Sathya et al., 2014)

In our study, we concluded that the transoral approach that had been applied in group I patients was more convenient to the operator and more accepted by the patients than the intraoral approach used in group III patients with its limited access and aggressive retraction as well as the extra-oral approach used in group II patients which needed more operative time and yielded a noticeable scar that was unaccepted to some patients included in the study. This was in agreement with (Ellis, 2010) who mentioned the complications of extraoral scar through which the $\mathrm{AO}$ reconstruction plates was inserted including the highest possibility of injury to the marginal mandibular branch of facial nerve.

Also, our study was in coincidence with other many previous studies that claimed; the more the conservative management through intra-oral approach was better because it avoids the high risk of injury of facial nerve, creating no facial scar as well as minimum post-operative pain and morbidity. (Barry and Kearns, 2007 ; Ellis , 2010 ; Hsueh et al., 2016 ; Pattar et al., 2014; Sudhakar et al ., 2015)

The strut plate allows for almost no movement at the superior and inferior borders with manual torsional and bending forces, as opposed to when a single linear plate is applied to the superior border area. When only 1 linear plate is placed at the superior border, torsional and bending forces usually exert movement along the axis of the plate with buccal-lingual splaying and formation of gap at the inferior border, respectively. (Guimond et al., 2005)

Because the screws are placed in a box configuration on both sides of the fracture rather than on a single line, broad platforms are created that may increase the resistance to torsional forces along the axis of the plate. The greater resistance to splaying of the inferior border with 2 plates as opposed to with a single plate at the superior border was clearly shown in the laboratory. (Zix et al., 2007)

This was in agreement to our study, we concluded that 3D plates in Group I showed no movement at the superior and inferior borders with manual torsional and bending forces, as opposed to Group III in which only single linear plate was applied to superior border, bending as well as torsional forces causes movement along the long axis of the plate with bucco-lingual splaying with formation of a gap at the inferior border.

Our study was also in coincidence with another study done by (Evagelos et al., 2009) who concluded that the $3 \mathrm{D}$ square plate system provided the most favorable mechanical behavior in comparison to the single and two miniplates.

Our study was in agreement with those of (Wittenberg et al., 1997) who worked on sheep mandibles and a Class III cantilever bending model, they found that the gap and displacement values for the angle $3 \mathrm{D}$ strut plate were comparable to 
those of the reconstruction plate. The infection rate $(5.4 \%)$ compares favorably with other studies using 2.0-mm systems for angle fractures, and most importantly, to the $7.5 \%$ infection rate reported with trans-cutaneously placed reconstruction plates.

(Scolozzi and Richter, 2003) concluded in their study that in cases of comminuted fractures, osteosynthesis should be performed in order to support full functional load as well as tensional forces redistribution while maintaining fractures fragments in its anatomic position. This is not possible by any technique, except $\mathrm{AO}$ reconstruction plate.

In our study, the healing phase passed uneventful regarding healing of bony segments and occlusion. In group $I$ as the screws were placed in box configuration on either side of fracture line in fractured segments rather than on single line. The broad platforms were created which may increase the resistance to the torsional forces along the axis of the plate. This was in coincidance with the results of (Guimond et al., 2005) in their study in treating mandibular angle fracture using the 3D strut plates. Also, agreed with (Wittenberg et al., 1997) as they stated that the gap and displacement values of the $3 \mathrm{D}$ curved strut plate were comparable to those of reconstruction plate.

In all cases teeth in fracture line were removed if they are fractured, infected, have gross caries, significant mobility, exposure of $50 \%$ or more of the root surface and/or interfere with fracture reduction.

The 3-dimensional miniplate used in the work by (Guimond et al., 2005) was installed with monocortical screws by intraoral approach; in neutral zone of mandibular angle region, there was less pressure on the plate against the bone surface and therefore less vascular rupture, thus reducing further complications. (Zix et al., 2007; Feledy et al.,2004)

Our results were in accordance with other studies which resulted low infection rate in correspondence with the use of the $3 \mathrm{D}$ plates such as those of
(Guimond et al., 2005) as they had infection rate of $5.4 \%$ ( 2 out of 37 patients) with the use of 3-D plates, also (Feledy et al., 2004) in their study had infection rate of $9 \%$ ( 2 out of 22 patients), and (Zix et al., 2007) who had $0 \%$ infection rate.

Also, in other studies where 3-dimensional plates were used, the result was low infection rate approximately $4.44 \%$ (2 patients) when compared with other study using conventional plates and miniplates, where the rate of infection in their result ranged from $3 \%$ to $32 \%$. (Feledy et al.,2004)

Our study was in agreement with study done by (Vivec et al., 2016) who concluded that the use of 3-D titanium miniplates system in mandibular anterior fractures was considered as a good alternative to Champy's 2 miniplates system as it provided good stability, lower rates of infection and need less intraoperative time compared to the regular miniplate systems.

Although our study was in agreement with a study done by (Yogesh et al.,2016) who proved that three dimensional plates was effective in the treatment of anterior mandibular fractures. Ease of its application and shorter working time were considered among its advantages over conventional miniplates.

In our study, in the comparison between percentage changes in infection rate of two groups, we proved that group III showed statistically significantly higher mean $\%$ increase than study group.

In the present study, pain intensity was significantly decreased in all cases of both groups across follow up period and this was in agreement with (Agarwal et al., 2014) who reported that a statistically significant difference was not found in the clinical parameters such as pain and swelling.

Also, (Melek et al., 2015) showed in their study that the postoperative clinical manifestations like pain and edema have resolved within the normal range of time in both groups and the findings were comparable between the two groups and within rea- 
sonable limits. Most probably adequate stabilization of the fracture segments that doesn't allow them to move apart eliminates the pain at the fracture site.

In the present study, maximum mouth opening was significantly increased in all cases of both groups across the follow-up period and this was in agreement with (Melek et al., 2015) who reported that patients of both groups had improvement in maximal mouth opening by time until they resumed normal mouth opening by the third postoperative month (35-40 mm).

In the present study, none of the patients in any of the groups had nonunion or plate fracture within the follow-up period and this was in agreement with (Agarwal et al., 2014) who reported that no such significant complications were noted in both conventional and 3D miniplates. Also, this was in acceptance with (Melek et al., 2015) who reported in their study on 3D plate in mandibular angle fracture.

\section{CONCLUSION}

The 3 Dimensional strut miniplates should be considered as a good successful alternative for internal rigid fixation of mandibular angle fractures for their superior advantages of ease of insertion transbuccally avoiding any extraoral cutaneous scars, lower infection rates, negligible possibility of facial nerve affection, while providing comparable segment stability and fixation rigidity that are achieved by reconstruction plates. Moreover, it obviates the need for immobilization of the mandible as required in Champy's technique.

\section{ACKNOWLEDGEMENT}

These two authors contributed equally.

\section{Conflict of interest:}

Authors have no conflict of interest.

\section{Funding}

This research had no funding source.

\section{Key finding / highlight}

3 D plates was considered an effective tool for open reduction and internal fixation in treatment of mandibular angle fracture with no complications.

\section{REFERENCES}

1. Agarwal M, Meena B, Gupta DK, Tiwari AD, Jakhar SK: A Prospective Randomized Clinical Trial Comparing 3D and Standard Miniplates in Treatment of Mandibular Symphysis and Parasymphysis Fractures. J Maxillofac Oral Surg 13: 79-83. https://doi.org/10.1007/s12663-0130483-x , 2014

2. Ahmed Gaber Hassanein: Trends and Outcomes of Management of Mandibular Fractures. J Craniofac Surg 30:12451251. https://doi.org/10.1097/SCS.0000000000005469, 2019

3. Barry CP, Kearns GJ: Superior border plating technique in the management of isolated mandibular angle fractures: a retrospective study. J Oral Maxillofac Surg 65:1544-9. https://doi.org/10.1016/j.joms.2006.10.069, 2007

4. Ellis E: A prospective study of three treatment methods for isolated fractures of the mandibular angle. J Oral Maxillofac Surg 68: 2743-54. https://doi.org/10.1016/j. joms.2010.05.080, 2010

5. Evagelos F Kalfarentzos, Despoina Deligianni, Georgios Mitros, Minos Tyllianakis: Biomechanical Evaluation of Plating Techniques for Fixing Mandibular Angle Fractures: The Introduction of a New 3D Plate Approach. Oral Maxillofac Surg 13:139-44. https://doi.org/10.1007/ s10006-009-0163-7 , 2009

6. Feledy J, Caterson EJ, Steger S: Treatment of mandibular angle fractures with a matrix miniplate: A preliminary report. Plast Reconstr Surg 114:1717-8. https://doi. org/10.1097/01.prs.0000142477.77232.f7,2004

7. Guimond C, Johnson JV, Marchena JM: Fixation of mandibular angle fractures with a 2.0-mm 3-dimensional curved angle strut plate. J Oral Maxillofac Surg 63:209-14. https://doi.org/10.1016/j.joms.2004.03.018,2005

8. Hsueh WD, Schechter CB, Tien Shaw I, Stupak HD: Comparison of intraoral and extraoral approaches to mandibular angle fracture repair with cost implications. Laryngoscope 126:591-5. https://doi.org/10.1002/lary. 25405, 2016 
9. Kubila S, Patiguli W, Bilikezi Y, Adili M:Three dimensional versus standard miniplate fixation in the management of mandibular fractures: A meta-analysis of randomized controlled trials. Kaohsiung Journal of Medical Sciences 33, 464. http://dx.doi.org/10.1016/j.kjms.2017.05.001 , 2017

10. Melek LN, El Mahallawy AS, Sharara AA, Ezz El Din A, Nour El-Din AHM. Evaluation of the 3-Dimensional Thread lock plate in the management of mandibular angle fractures. Tanta Dental Journal 12:140-148. https://doi. org/10.1016/j.tdj.2015.05.001, 2015

11. Omezli MM, Ayranci F, Polat ME, Dayi E, Ghahraman E zadehasl, Karagol S: Biomechanical Comparison of Transoral and Transbuccal Lateral Cortical Plate Fixation for the Management of Mandibular Angle Fractures. Nigerian Journal of Clinical Practice, 20:1434-8. https://doi. org/10.4103/1119-3077.187319,2017

12. Pattar P, Shetty S, Degala S: A prospective study on management of mandibular angle fracture. J Maxillofac Oral Surg 13:592-8. https://doi.org/10.1007/s12663-013-0542-3, 2014

13. Ravikumar C, Bhoj M: Evaluation of postoperative complications of open reduction and internal fixation in the management of mandibular fractures: A retrospective study. Indian J Dent Res 30:94-96. https://doi.org/10.4103/ ijdr.IJDR_116_17, 2019

14. Sathya Kumar Devireddy, R.V. Kishore Kumar, Rajasekhar Gali, Sridhar Reddy Kanubaddy, Mallikarjuna Rao Dasari, and Mohammad Akheel: Transoral versus extraoral approach for mandibular angle fractures: A comparative study. Indian J Plast Surg 47: 354-361 . https://doi. org/10.4103/0970- 0358.146590 . 2014
15. Scolozzi P, Richter M: Treatment of severe mandibular fractures using AO reconstruction plates. J Oral Maxillofac Surg 61: 458-61. https://doi.org/10.1053/joms.2003.50087, 2003

16. Sudhakar GV, Rajasekhar G, Dhanala S, Vura N, Ramisetty S: Comparison of management of mandibular angle fractures by three approaches. J Maxillofac Oral Surg 14: 979-85. https:// doi.org/10.1007/s12663-015-0779-0, 2015.

17. Thomas A., Chiodo, Maano Milles: Use of Monocortical Miniplates for the Intraoral Treatment of Mandibular Fractures. Atlas Oral Maxillofacial Surg Clin N Am 17: 19-25. https://doi.org/10.1016/j.cxom.2008.10.008 , 2009

18. Vivek GK, Akshay Shetty, N. Vaibhav, Mohammad Imran: A comparative study of single 3-D titanium plate versus conventional Champy's 2 miniplate fixation in the management of mandibular anterior fracture: A prospective clinical study. Journal of Advanced Clinical \& Research Insights , 3: 194199. https://doi.org/10.15713/ins.jcri.137, 2016.

19. Wittenberg JM, Mukherjee DP, Smith BR: Biomechanical evaluation of new fixation devices for mandibular angle fractures. Int J Oral Maxillofac Surg 26:68-73. https://doi. org/10.1016/s0901-5027(97)80852-1 , 1997.

20. Yogesh Mittal, George Varghese, S. Mohan, N. Jayakumar, Somil Chhag: A Comparative Study of 3-Dimensional Titanium Versus 2-Dimensional Titanium Miniplates for Open Reduction and Fixation of Mandibular Parasymphysis Fracture. J. Maxillofac. Oral Surg 15:93-98 https://doi. org/10.1007/s12663-015-0780-7, 2016.

21. Zix J, Lieger O, Iizuka T: Use of straight and curved 3-dimensional titanium miniplates for fracture fixation at the mandibular angle. J Oral Maxillofac Surg 65:1758-63. https://doi.org/10.1016/j.joms.2007.03.013, 2007. 